\title{
Tony Halliday, The Temperamental nude: class, medicine and representation in eighteenth-century
}

\author{
France
}

\section{Vittorio Fortunati}

\section{(2) OpenEdition}

\section{Journals}

\section{Edizione digitale}

URL: http://journals.openedition.org/studifrancesi/5625

DOI: $10.4000 /$ studifrancesi.5625

ISSN: 2421-5856

\section{Editore}

Rosenberg \& Sellier

\section{Edizione cartacea}

Data di pubblicazione: 1 septembre 2011

Paginazione: 408

ISSN: 0039-2944

\section{Notizia bibliografica digitale}

Vittorio Fortunati, «Tony Halliday, The Temperamental nude: class, medicine and representation in eighteenth-century France», Studi Francesi [Online], 164 (LV | II) | 2011, online dal 30 novembre 2015, consultato il 12 janvier 2021. URL: http://journals.openedition.org/studifrancesi/5625 ; DOI: https:// doi.org/10.4000/studifrancesi.5625

Questo documento è stato generato automaticamente il 12 janvier 2021.

\section{cc) (†) $\odot$}

Studi Francesi è distribuita con Licenza Creative Commons Attribuzione - Non commerciale - Non opere derivate 4.0 Internazionale. 


\title{
Tony Halliday, The Temperamental nude: class, medicine and representation in eighteenth-century
} France

\author{
Vittorio Fortunati
}

\section{NOTIZIA}

TONY HALLIDAY, The Temperamental nude: class, medicine and representation in eighteenthcentury France, Oxford, Voltaire Foundation, 2010, $257 \mathrm{pp}$.

1 Per molti secoli le differenze di aspetto e di carattere fra gli individui vennero spiegate in base alla ben nota teoria dei quattro umori. La credibilità di quest'ultima fu messa irrimediabilmente in crisi fin dagli albori della rivoluzione scientifica del xvII secolo, senza che per questo scomparisse la tendenza a vedere nei tratti somatici i segni esteriori della personalità morale e psicologica. A ciò probabilmente si deve, nel secolo successivo, l'elaborazione di una nuova teoria dei temperamenti, caratterizzata da importanti valenze sociologiche, la quale ebbe tra i suoi maggiori esponenti prima Albrecht von Haller, più tardi Johann Kaspar Lavater e Pierre Jean Georges Cabanis. La riflessione di questi studiosi ebbe notevoli ripercussioni anche fuori dall'ambito prettamente scientifico, influenzando la rappresentazione del corpo umano (in particolare del nudo maschile) nelle arti figurative europee del xvIII secolo. T. Halliday, storico dell'arte scomparso nel 2006, noto soprattutto per i suoi lavori sul genere del ritratto e sull'arte francese negli anni della Rivoluzione, si è concentrato sugli effetti della teoria dei temperamenti sulla pittura e la scultura nella Francia del tardo Settecento, con una particolare attenzione per il periodo rivoluzionario e postrivoluzionario. 
2 È appunto nei capitoli dedicati a quel cruciale momento storico che sono contenuti, a nostro avviso, i risultati più originali della ricerca che sta alla base del volume in oggetto. Ci riferiamo, per esempio, alle pagine che descrivono il dibattito, accesosi negli anni del momentaneo trionfo dei rivoluzionari, intorno al modo di rappresentare artisticamente il popolo francese, inteso come insieme dei cittadini del nuovo regime: l'A. sottolinea come la scelta, a quello scopo, del nudo idealizzato corrisponda all'interclassismo dell'ideologia giacobina («A body one and indivisible: Jacobin representation of the people», pp. 155-188). Di grande interesse è, inoltre, l'analisi del celebre quadro di Jacques-Louis David che rappresenta il combattimento fra romani e sabini; secondo l'A., i corpi nudi di Romolo e Tito Tazio raffigurano, rispettivamente, i due temperamenti opposti descritti da Cabanis: il «cerebrale» e il «muscolare», l'uno dominato dalla ragione, l'altro dall'istinto e dalle passioni ( $«$ The trouble with Tatius: David's Sabines and the end of public art», pp. 221-236).

3 The Temperamental nude non è solo un valido saggio di storia dell'arte, ma fornisce numerosi spunti di indagine a tutti coloro che si occupano, sotto vari aspetti, della cultura francese settecentesca: anche per questo ci è parso doveroso segnalarlo sulla nostra rivista. 